\title{
Performance Improvement of Broadband Distributed Raman Amplifier Using Bidirectional Pumping with First and Dual Order Forward Pumps
}

\author{
Md A. Iqbal ${ }^{1}$, Giuseppe R. Martella ${ }^{2}$, Francesca Gallazzi ${ }^{2}$, Mingming Tan $^{1}$, Paul Harper $^{1}$, Juan Diego \\ Ania-Castañón ${ }^{2}$ \\ ${ }^{1} A I P T$, Aston University, Birmingham B4 7ET, UK \\ ${ }^{2}$ Instituto de Óptica "Daza de Valdés”, CSIC, C/ Serrano 121, 28006 Madrid, Spain' \\ Tel: (+44) 121204 3512, e-mail: iqbalm7@aston.ac.uk
}

\begin{abstract}
In this paper, a new bidirectional pumping scheme with dual order forward pumps is proposed. Performance is compared numerically with conventional bidirectional and backward only pumping schemes for a $70 \mathrm{~nm}$ bandwidth, $61.5 \mathrm{~km}$ distributed Raman amplifier. We demonstrate that it is possible to design a flat gain spectrum with improved noise figure and OSNR, as well as a low gain ripple $(<1 \mathrm{~dB})$.
\end{abstract}

Keywords: broadband Raman amplification, bidirectional pumping, noise figure.

\section{INTRODUCTION}

The general purpose of multi-wavelength pump Raman amplifier is to provide a broad gain bandwidth with a very flat gain profile [1]. Backward pumping is usually preferred in multi-wavelength pumping because of its superior performance over forward pumping in terms of relative intensity noise (RIN) transfer from pump to signal and gain saturation threshold [2,3]. Larger values of noise figure and tilt are the fundamental problems of using multi-wavelength backward only pumping scheme [4]. Y. Emori et al. first introduced the idea of optimized bidirectional pumping in broadband Raman amplifiers using shortest wavelength pumps for forward pumping to realize flat gain and optical noise figure (NF) spectrum [5]. It is very important to use forward pumps with low RIN values (e.g. semiconductor pump lasers) because data transmission performance can be highly degraded by the impacts of RIN transfer at higher pump powers [6]. In order to achieve a low intra-span signal power variation, higher or dual order pumping can be used to distribute the gain more evenly along the span, leading to better optical signal to noise ratio (OSNR) and noise figure (NF) performance [7-9]. The benefits of higher order pumping have also been demonstrated in $[10,11]$ in terms of extended reach of data transmission where transmission bandwidth was limited in C-band.

Here we propose a new bidirectional pumping scheme for broadband Raman amplifier which includes both dual and shortest first order forward pumps to achieve better OSNR and reduced optical NF of WDM signals.

\section{PROPOSED SCHEMES AND NUMERICAL MODEL}

Three different pumping schemes were investigated as shown in Figure 1. In all cases, five backward pump wavelengths were used: $1425 \mathrm{~nm}, 1444 \mathrm{~nm}, 1462 \mathrm{~nm}, 1476 \mathrm{~nm}$ and $1508 \mathrm{~nm}$. This combination of wavelengths gives a flat gain profile over a broad signal bandwidth from 1530 to $1610 \mathrm{~nm}$ for a $61.5 \mathrm{~km}$ amplifier span. In Figure 1(b) the shortest wavelength pump at $1425 \mathrm{~nm}$ has been used as a forward pump to improve the OSNR and NF of shorter wavelength signals. In Figure 1(c), $2^{\text {nd }}$ order forward Raman pumping has been considered: both $1365 \mathrm{~nm}$ and $1425 \mathrm{~nm}$ pump wavelengths are used, with the $1425 \mathrm{~nm}$ pump acting as a seed which is amplified by the $1365 \mathrm{~nm}$ pump and finally amplifies shorter wavelength signals in order to improve the ASE noise performance of the amplifier.

The full numerical model for the evolution of WDM pumps and signals is based on the standard model presented in $[7,8]$ and also extended for OSNR evolution and optical NF calculation at signal wavelengths. All important effects such as stimulated and spontaneous Raman scattering, pump depletion, ASE and double Rayleigh scattering (DRS) noise, energy transfer due to pump-pump, pump-sig and sig-sig interactions from either directions are included in the model and described in the following equation:

$$
\frac{d P_{v}^{ \pm}}{d z}= \pm\left\{\begin{array}{l}
-\alpha_{v} P_{v}^{ \pm}+\varepsilon_{v} P_{v}^{\mp} \\
+P_{v}^{ \pm}\left[\sum_{\mu>\nu} \frac{g_{\mu v}}{A_{\mu}}\left(P_{\mu}^{+}+P_{\mu}^{-}\right)-\sum_{\mu<\nu} \frac{v}{\mu} \frac{g_{v \mu}}{A_{\mu}}\left(P_{\mu}^{+}+P_{\mu}^{-}\right)-4 h v \sum_{\mu<v} \frac{g_{v \mu}}{A_{\mu}}\left(1+\frac{1}{e^{\frac{h(v-\mu)}{k T}}-1}\right) \Delta \mu\right. \\
+2 h v \Delta v \sum_{\mu>v} \frac{g_{\mu \nu}}{A_{\mu}}\left(P_{\mu}^{+}+P_{\mu}^{-}\right)\left(1+\frac{1}{e^{\frac{h(\mu-v)}{k T}}-1}\right)
\end{array}\right\}
$$


where $P^{ \pm}$represents the power within the frequency interval $\Delta v$ either forward (+) or backward (-) propagating direction at centre frequency $v . \alpha_{v}$ and $\varepsilon_{v}$ are the fiber attenuation and Rayleigh scattering coefficient at frequency $v$ respectively. $g_{\mu \nu}$ and $A_{\mu}$ represent the Raman gain coefficient at frequency $v$ due to pump at frequency $\mu$ and effective core area of fiber at frequency $\mu$ respectively, $h$ is the Plank's constant, $k$ is the Boltzmann's constant and $T$ is the absolute temperature. The above model has been extended as following to get corresponding noise power evolution at frequency $v$ :

$$
\begin{aligned}
& \frac{d N_{v}^{+}}{d z}=-\alpha_{v} N_{v}^{+}+\varepsilon_{v} N_{v}^{-}+N_{v}^{+} \sum_{i=1}^{N_{P}} \frac{g_{\mu i v}}{A_{\mu i}}\left(P_{\mu i}^{+}+P_{\mu i}^{-}\right)+2 h v \Delta v \sum_{i=1}^{N_{P}} \frac{g_{\mu i v}}{A_{\mu i}}\left(P_{\mu i}^{+}+P_{\mu i}^{-}\right)\left(1+\frac{1}{e^{\frac{h(\mu i-v)}{k T}}-1}\right) \\
& \frac{d N_{v}^{-}}{d z}=\alpha_{v} N_{v}^{-}-\varepsilon_{v}\left(N_{v}^{+}+P_{S}(v)\right)-N_{v}^{-} \sum_{i=1}^{N_{P}} \frac{g_{\mu i v}}{A_{\mu i}}\left(P_{\mu i}^{+}+P_{\mu i}^{-}\right)-2 h v \Delta v \sum_{i=1}^{N_{P}} \frac{g_{\mu i v}}{A_{\mu i}}\left(P_{\mu i}^{+}+P_{\mu i}^{-}\left(1+\frac{1}{e^{\frac{h(\mu i-v)}{k T}}-1}\right)\right.
\end{aligned}
$$

where $N^{ \pm}$denotes the average noise power in forward (+) or backward (-) propagating direction at the signal frequency $v, N_{P}$ and $A_{\mu}$ are the number of pump frequencies and effective fiber core area at the $i^{\text {th }}$ pump frequency $\mu i . P_{S}(v)$ and $g_{\mu i v}$ represent average signal power at frequency $v$ and Raman gain coefficient from $i^{\text {th }}$ pump at frequency $\mu i$ to noise frequency $v$.

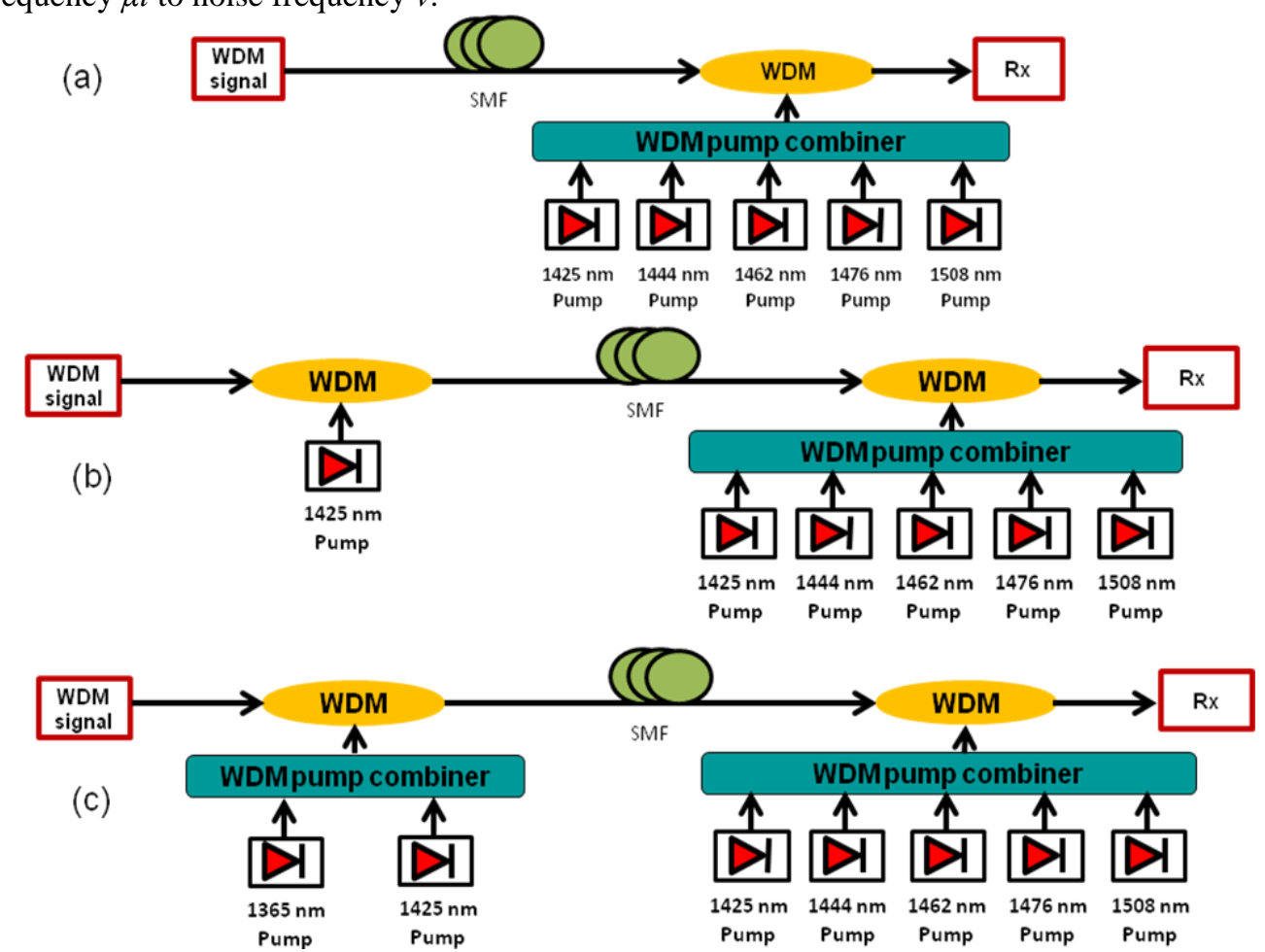

Figure 1: Schematic depiction of the proposed three schemes: (a) scheme-1: backward only pumping with 5 pumps (b) scheme-2: bidirectional pumping with shortest wavelength pump as forward pump and (c) scheme-3: bidirectional pumping with $1365 \mathrm{~nm} 2^{\text {nd }}$ order pump and $1425 \mathrm{~nm}$ pump seed from input end

Numerical simulations have been carried out considering room temperature, completely depolarised pumps to neglect the polarisation dependence of Raman gain and $125 \mathrm{GHz}$ of noise bandwidth. Raman gain and attenuation coefficients at different frequencies have been chosen with respect to the normalized Raman gain spectrum due to depolarized pumps and attenuation profile of standard SMF silica fiber. Eight equally spaced signal channels $(1530 \sim 1600 \mathrm{~nm})$ have been considered with $-10 \mathrm{dBm}$ power per channel. Table 1 shows the corresponding pump powers used in numerical simulation in different schemes. The equivalent noise figure at a signal frequency $v$ has been calculated using the following standard expression [12]:

$$
\begin{aligned}
& N F=\frac{P_{A S E}}{E_{p h} B_{0} G}+\frac{1}{G} \\
& N F(d B)=10 \log 10(N F)
\end{aligned}
$$

where $P_{A S E}$ and $E_{p h}$ are the ASE noise power and photon energy at frequency $v . G$ takes the on-off gain value and $B_{0}$ is the reference optical bandwidth. 
Table 1. Pump powers used in numerical simulation.

\begin{tabular}{|c|c|c|c|c|c|}
\hline Pumps & Scheme-1 & \multicolumn{2}{|c|}{ Scheme-2 } & \multicolumn{2}{c|}{ Scheme-3 } \\
\cline { 2 - 6 } & $\begin{array}{c}\text { (Backward Only) } \\
(\mathrm{mW})\end{array}$ & $\begin{array}{c}\text { Forward } \\
(\mathrm{mW})\end{array}$ & $\begin{array}{c}\text { Backward } \\
(\mathrm{mW})\end{array}$ & $\begin{array}{c}\text { Forward } \\
(\mathrm{mW})\end{array}$ & $\begin{array}{c}\text { Backward } \\
(\mathrm{mW})\end{array}$ \\
\hline $1365 \mathrm{~nm}$ & - & - & - & 500 & - \\
\hline $1425 \mathrm{~nm}$ & 258.8 & 50 & 250 & 10 & 90 \\
\hline $1444 \mathrm{~nm}$ & 191 & - & 191 & - & 100 \\
\hline $1462 \mathrm{~nm}$ & 88.3 & - & 88.3 & - & 80 \\
\hline $1476 \mathrm{~nm}$ & 82.8 & - & 82.8 & - & 105 \\
\hline $1508 \mathrm{~nm}$ & 99.8 & - & 99.8 & - & 160 \\
\hline
\end{tabular}

\section{RESULTS AND DISCUSSION}

All the results have been obtained through numerical simulation according to the model described in section 2 . Performance of our proposed new scheme-3 have been compared to other two traditional schemes in terms of output OSNR, optical equivalent noise figure and signal power evolution of a specific signal wavelength at 1530 nm.

(a)

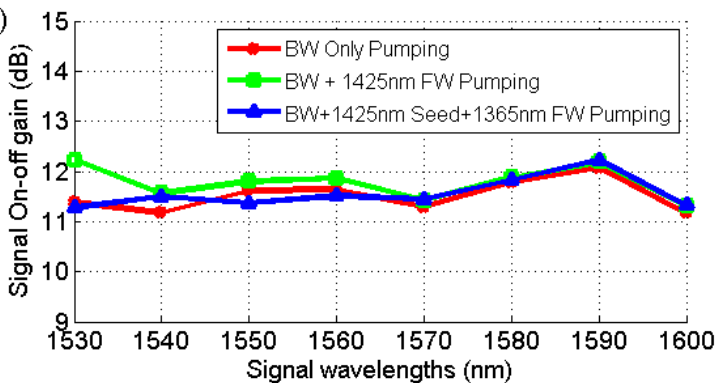

(b)

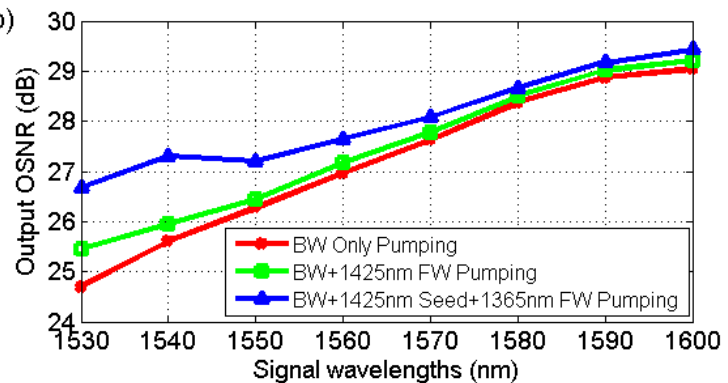

Figure 2. Comparison of (a) On-off gain in $d B$ and (b) output OSNR in $d B$ among three different schemes for $61.5 \mathrm{~km}$ broadband distributed Raman amplifier span

Figure 2(a) shows the on-off gain spectra of three different schemes. Pump powers have been optimized in each cases to have similar average on-off gain about $11.5 \sim 11.8 \mathrm{~dB}$ in order to carry out a fair comparison in terms of OSNR and optical NF in the whole amplifier bandwidth. Figure 2(b) shows that the output OSNR improves as we go from backward only pumping to bidirectional pumping and our proposed scheme-3 gives the best output OSNR values over the whole $70 \mathrm{~nm}$ bandwidth that has been considered in the numerical simulations.
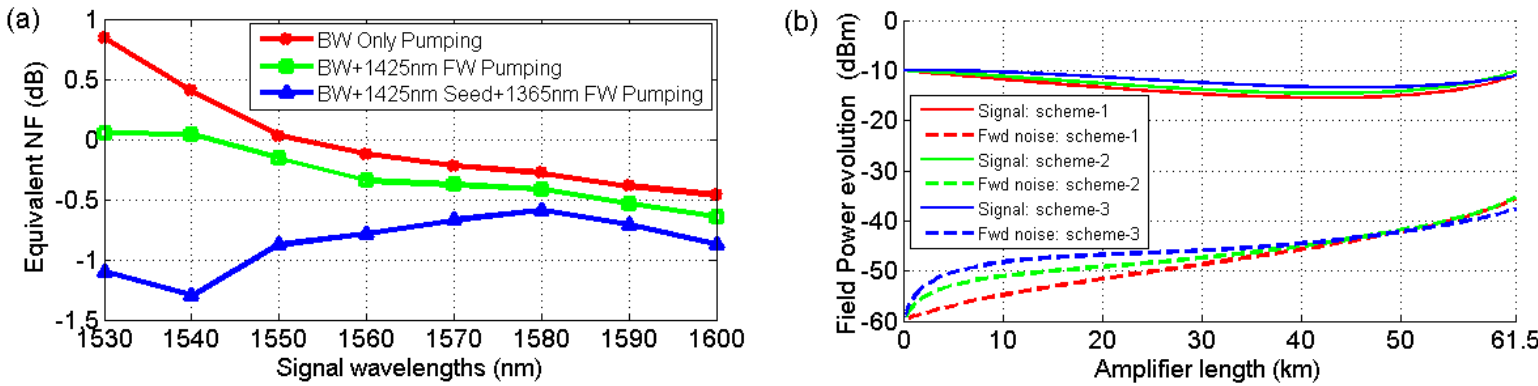

Figure 3 (a) Comparison of equivalent $N F$ in $d B$ and $(b)$ Field power evolution of signal and forward propagating noise at $1530 \mathrm{~nm}$ among three different schemes

Figure 3(a) shows equivalent NF of different signal channels for different schemes. Backward only pumping has the highest NF tilt, as expected, where lower wavelength signals suffer the most. The overall NF tilt or variation has been reduced in scheme-2 and 3. Scheme-3 performs the best giving lowest NF values in all signal wavelengths. This is due to an improved SPV along the span and minimization of ASE noise build up as shown in Figure 3(b).

Table 2 summarises the characteristics of the three schemes tested. There was a slight increase in the gain ripple for scheme-3 compared to the other two schemes but more importantly scheme-3 had the lowest value of equivalent NF and total ASE noise power, the best SPV and output OSNR and flatter NF spectrum compared to other cases. A full transmission performance analysis including RIN is required to confirm the performance of 
this amplification scheme but the results presented here indicate the potential to simultaneously improve performance compared to conventional backward only pumping and increase gain bandwidth beyond the traditional C-band.

Table 2. Comparison of different features.

\begin{tabular}{|l|c|c|c|}
\hline Features & Scheme-1 & Scheme-2 & Scheme-3 \\
\hline Gain ripple (dB) & 0.91 & 0.92 & 0.95 \\
\hline Equivalent NF variation (dB) & 1.3 & 0.7 & 0.7 \\
\hline Equivalent NF value (dB) @ 1530 nm & 0.84 & 0.05 & -1.10 \\
\hline SPV (dB) @ 1530 nm & 5.5 & 4.6 & 3.4 \\
\hline Total ASE noise power (dBm) @ 1530 nm & -35.6 & -35.5 & -37.7 \\
\hline Output OSNR (dB) @ 1530 nm & 24.7 & 25.4 & 26.7 \\
\hline
\end{tabular}

\section{CONCLUSIONS}

We have investigated numerically the improvement in OSNR and NF performance of our proposed bidirectionally pumped broadband distributed Raman amplifier which uses a combination of $1^{\text {st }}$ order backward pumping and $2^{\text {nd }}$ order forward pumping wavelengths in combination with backward only pumping schemes. In order to avoid the deleterious effect of RIN transfer from the forward pumps, the $2^{\text {nd }}$ order $1365 \mathrm{~nm}$ pump should, ideally, be a low-RIN laser (e.g. semiconductor-based), but the better noise performance of the proposed scheme has the potential to improve transmission performance in high capacity broadband Raman amplified systems.

\section{ACKNOWLEDGEMENTS}

This work was funded by FP7 ITN programme ICONE (608099).

\section{REFERENCES}

[1] S. Namiki, Y. Emori: Ultrabroad-band Raman amplifiers pumped and gain-equalized by wavelengthdivision multiplexed high-power laser diodes, IEEE J. Sel. Topics In Quant. Electr., vol. 7. no. 1. pp. 3-16, Jan/Feb. 2001.

[2] C. R. S. Fludger, V. Handerek, R. J. Mears: Pump to signal RIN transfer in Raman fiber amplifiers, J. Lightwave Technol., vol. 19. no. 8. pp. 1140-1148, Aug. 2001.

[3] K. Song, S.D. Dods: Cross modulation of pump-signals in distributed Raman amplifiers, theory and experiment, IEEE Photon. Technol. Lett., vol. 13. no. 11. pp. 1173-1175, Nov. 2001.

[4] C. R. S. Fludger, V. Handerek, R. J. Mears: Fundamental noise limits in broadband Raman amplifiers, in Proc. OFC 2001, Anaheim, CA, USA, Mar. 2001, paper MA5.

[5] S. Kado, Y. Emori, S. Namiki, N. Tsukiji, J. Yoshida, T. Kimura: Broadband flat-noise Raman amplifier using low-noise bidirectionally pumping sources, in Proc. ECOC 2001, Amsterdam, Netherlands, Sept. 2001, paper PD.F.1.7.

[6] M. Tan, P. Rosa, S. Le, I. Phillips, P. Harper: Evaluation of 100G DP-QPSK long-haul transmission performance using second order co-pumped Raman laser based amplification, Opt. Express, vol. 23. pp. 22181-22189, 2015.

[7] J. Ania-Castañón: Quasi-lossless transmission using second-order Raman amplification and fibre Bragg gratings, Opt. Express, vol. 12. no. 19. pp. 4372-4377, 2004.

[8] J. D. Ania-Castañón, A. A. Pustovskikh, S. M. Kobtsev, S. K. Turitsyn: Simple design method for gainflattened three-pump Raman amplifiers, Opt. and Quantum Electron., vol. 39, no. 3. pp. 213, 2007.

[9] M. Tan, P. Rosa, I. D. Phillips, P. Harper: Long-haul Transmission Performance Evaluation of Ultra-long Raman Fiber Laser Based Amplification Influenced by Second Order Co-pumping," in Asia Communications and Photonics Conference 2014, OSA Technical Digest (online) (Optical Society of America, 2014), paper ATh1E.

[10] S. Faralli, G. Bolognini, G. Sacchi, S. Sugliani, F. Di Pasquale: Bidirectional higher order cascaded Raman amplification benefits for 10-Gb/s WDM unrepeated transmission systems, J. Lightwave Technol., vol. 23. no. 8. pp. 2427-2433, Aug. 2005.

[11] M. Tan et al.: Transmission comparison of ultra-long Raman fibre laser based amplification with first and dual order Raman amplification using 10×118 Gbit/s DP-QPSK, in Proc. ICTON 2014, Graz, Jul. 2014, paper Tu.C1.7.

[12] W. Zhang, J. Peng, X. Liu, C. Fan: An analytical expression of equivalent noise figure for distributed fiber Raman amplifiers with Rayleigh scattering, Optics communications, vol. 199. no. 1. pp. 231-236, Nov. 2001. 